\title{
CONOCIMIENTO Y APLICACIÓN DE LOS PRINCIPIOS BIOÉTICOS EN ALUMNOS DE POSGRADO DE ODONTOLOGÍA
}

\author{
Gabriela Morales¹, Ramiro Ramadán², Susana Alvear D², Galo Sánchez-del-Hierro² \\ Resumen: La bioética ha sido agregada formalmente, desde 2008, a la malla curricular de la carrera de Odontología en el \\ Ecuador. También se han creado documentos que rigen el accionar ético del odontólogo frente a distintos dilemas que se \\ pueden presentar durante su actividad clínica. Esta investigación busca describir el nivel del conocimiento y la aplicación de \\ los principios bioéticos en los profesionales de salud oral. Se utilizó un método de investigación cuantitativo, descriptivo y \\ transversal, mediante una encuesta estructurada, aplicada a estudiantes de posgrado en el área odontológica. Los datos fueron \\ procesados y analizados de forma descriptiva y correlacional. Los resultados obtenidos, resaltaron la presencia de cinco niveles \\ de conocimiento sobre principios bioéticos. Entre estos, en los grupos de mayor porcentaje de conocimiento sobre los principios \\ bioéticos se destacó la aplicación del principio de no maleficencia en su práctica clínica. Se reveló desconocimiento sobre la \\ teoría: muchos desconocen los principios bioéticos básicos del correcto accionar del profesional de salud oral. Sin embargo, \\ se ha demostrado un creciente cambio con respecto a la aplicación de estos principios.
}

Palabras clave: bioética, odontología, principios bioéticos, conocimiento, beneficencia, no maleficencia, justicia, autonomía

\section{Knowledge and application of bioethical principles in postgraduate Dental Students}

\begin{abstract}
Bioethics has been formally added, since 2008, to the curricula of dentistry courses in Ecuador. Documents have also been created that govern the ethical actions of the dentist in the face of different dilemmas that may arise during their clinical activity. This research seeks to describe the level of knowledge and application of bioethical principles in oral health professionals. A quantitative, descriptive and cross-sectional research method was used, by means of a structured survey, applied to postgraduate students in the dental area. The data were processed and analyzed in a descriptive and correlational manner. The results obtained highlighted the presence of five levels of knowledge of bioethical principles. Among these, in the groups with the highest percentage of knowledge about bioethical principles, the application of the principle of non-maleficence in their clinical practice was highlighted. Lack of knowledge about the theory was revealed: many are unaware of the basic bioethical principles of the correct action of the oral health professional. However, a growing change has been demonstrated with regard to the application of these principles.
\end{abstract}

Keywords: bioethics, dentistry, bioethical principles, knowledge, beneficence, non maleficence, justice, autonomy

\section{Conocimiento y aplicación de los principios bioéticos en alumnos de posgrado de Odontología}

Resumo: A bioética tem sido formalmente acrescentada ao currículo dos cursos de odontologia no Equador desde 2008. Também foram criados documentos para reger as açóes éticas dos dentistas quando confrontados com diferentes dilemas que podem surgir durante sua atividade clínica. Esta pesquisa procura descrever o nível de conhecimento e aplicação dos princípios bioéticos nos profissionais de saúde bucal. Foi utilizado um método de pesquisa quantitativa, descritiva e transversal, por meio de uma pesquisa estruturada aplicada a estudantes pós-graduados na área odontológica. Os dados foram processados e analisados de forma descritiva e correlacional. Os resultados obtidos destacaram a presença de cinco níveis de conhecimento dos princípios bioéticos. Entre estes, nos grupos com maior porcentagem de conhecimento de princípios bioéticos, foi destacada a aplicação do princípio de não maleficência na prática clínica. A ignorância da teoria foi revelada: muitos desconhecem os princípios básicos de bioética da açáo correta do profissional de saúde oral. No entanto, uma mudança crescente tem sido demonstrada com relação à aplicaçáo destes princípios.

Palavras-chave: bioética, odontologia, princípios bioéticos, conhecimento, beneficência, não maleficência, justiça, autonomia

\footnotetext{
${ }^{1}$ Pontificia Universidad Católica del Ecuador (PUCE), Ecuador. Facultad de Odontología de la Universidad Central del Ecuador, Ecuador. ORCID: 0000-0001-5537-3232

Correspondencia: ggmorales@puce.edu.ec

${ }^{2}$ Facultad de Medicina de la Pontificia Universidad Católica del Ecuador (PUCE), Ecuador.
} 


\section{Introducción}

La práctica del profesional en salud bucodental no debe estar separada de la reflexión moral. Cuando la relación dentista-paciente es de confianza mutua, las situaciones inusuales y difíciles son más fáciles de resolver. Para generar confianza en sus pacientes, el odontólogo requiere seguir pautas morales que ofrezcan una consideración específica al presentar opciones de tratamiento, obtener el consentimiento informado y responder a las decisiones de los pacientes(1). El odontólogo no debe ser ajeno a los fundamentos, valores, principios y metodologías que la bioética propone $(2,3)$. Por este motivo se imparte dentro de la formación académica de los futuros profesionales de esta área y la enseñanza en bioética debe ser vista como un elemento preventivo, que permite transformar la práctica de las ciencias de la salud con un enfoque hacia valores sociales $(2,4)$. Los odontólogos se ven en la responsabilidad de reconocer claramente sus deberes resumidos en: saber, saber hacer y saber cuándo hacer, camino que lleva al futuro odontólogo a actuar con prudencia, que es la virtud máxima de las profesiones(5).

La Asociación Dental Americana (ADA) ha incluido entre estos principios a la veracidad, que se puede dedefinir como la obligación de responder a esa posición de confianza inherente frente al paciente, mejorando la relación con el mismo, comunicándose sin engaños y manteniendo la integridad intelectual(6). El principio de la veracidad se suele plasmar en la comunicación entre profesional y paciente, con un lenguaje comprensible y probabilidades sobre las actividades que se llevarán a cabo en la práctica clínica(7).

En Latinoamérica existe un porcentaje mínimo de estudios que remarcan el rol de la bioética en la odontología(8); sin embargo, se ha logrado promover una praxis enfocada en los principios bioéticos, que tiene como objetivo principal el bienestar del paciente. Una aplicación clínica más consciente, en la que se vea al paciente como la persona en la que se ejercerán conocimientos para lograr un adecuado estado de salud oral(2).

Se ha concluido que el profesional odontológico latinoamericano debe trabajar en conjunto, para elevar las cifras sobre aplicaciones bioéticas que contribuyan a enaltecer a la profesión y le permitan llevar sus diagnósticos, planes de tratamiento, cobros, guías y procedimientos clínicos con una mayor responsabilidad social(9). En Latinoamérica se ha transformado en un desafío la relativa debilidad de los programas y contribuciones en bioética dentro del campo de la salud oral; no solamente en los odontólogos dedicados a la práctica clínica, sino también en planificadores y docentes en las universidades, que carecen de un enfoque sistemático a los grandes temas bioéticos que se desarrollan en el transcurso de la práctica profesional(10).

Ecuador ha sumado "bioética" como cátedra dentro de la malla curricular de la carrera de pregrado en todas las instituciones que ofrecen educación en el área odontológica. Se han formulado documentos de alta relevancia, cuya finalidad es salvaguardar la dignidad de la profesión(11). En 2008 se presenta la "Declaración de Quito sobre Bioética y Salud Oral", en la que se sostiene la invariable adhesión de la odontología a los todos principios remarcados en aquellos documentos. Este documento fue guía en la creación del "Código Ecuatoriano de Ética y Deontología Odontológica" (CEEDO), basado en los principios bioéticos de justicia, beneficencia, no maleficencia y autonomía(11).

Esta investigación tuvo como objetivo conocer el nivel de los conocimientos y la aplicación de los principios bioéticos de los estudiantes de posgrado en el área odontológica.

\section{Materiales y métodos}

Se trató de una investigación cuantitativa, descriptiva, transversal. Se aplicó la encuesta de dieciocho preguntas a sesenta y ocho estudiantes inscritos en los programas de posgrado de la Universidad Central del Ecuador, esperando una tasa de respuesta del $60 \%$.

El instrumento fue estructurado para esta investigación, calibrado y valorado por expertos en el área metodológica, bioética y odontológica. La encuesta está dividida en tres grupos; el primero con siete preguntas, destinadas a la descripción de los participantes; un segundo grupo compuesto por cinco preguntas que contribuyeron a iden- 
tificar el nivel de conocimiento teórico de los participantes sobre los principios bioéticos; y un tercer grupo de seis preguntas relacionadas con casos clínicos encaminados a reconocer la aplicación de los principios de justicia, beneficencia, no maleficencia y autonomía en la práctica clínica. Los datos obtenidos fueron tabulados y estratificados por medio de valores porcentuales a los participantes. Se realizó un análisis descriptivo y de correlación de acuerdo con las variables por medio de t-student y Chi-cuadrado. Se utilizó un programa para el análisis de datos de muestreo en sistema GNU pspp 1.4.1-g79ad47.

Este estudio fue aprobado por el Comité de Ética de la Investigación de la Pontificia Universidad Católica del Ecuador (Of No CEI-96.2020); se obtuvo el permiso de las autoridades del Instituto Superior de Investigación de Posgrado de la Facultad de Odontología, Universidad Central del Ecuador; y el consentimiento informado de cada participante.

\section{Resultados}

Cincuenta y cuatro participantes $(79,4 \%)$ respondieron la encuesta. El 72,2\% ( $\mathrm{n}=39)$ de los participantes del estudio es de sexo masculino, la mayoría pertenecía al programa de formación de posgrado de Estética y Operatoria en un 20,4\% $(\mathrm{n}=11)$; un $83,3 \%(\mathrm{n}=45)$ recibió la cátedra de bioética dentro de su formación de pregrado, mientras que un 7,4\% $(n=4)$ recibió adicionalmente un curso formal en bioética. El 19,6\% $(\mathrm{n}=11)$ de los estudiantes afirmó conocer el CEEDO.

El 43,5\% ( $n=23)$ de los estudiantes encuestados reconoció los cuatro principios bioéticos en los que se basa el CEEDO; el 40,\% (n=22) identificó a la veracidad como el quinto principio bioético de la conducta profesional; únicamente el 13\% $(\mathrm{n}=7)$ señaló al Informe Belmont como el documento histórico que destacó los principios y las pautas para la protección de seres humanos en la investigación; El 70,4\% ( $\mathrm{n}=38)$ reconoció el respeto a la dignidad humana como el principio bioético más elevado en las prácticas sanitarias, mientras que el 40,7\% ( $\mathrm{n}=22)$ identificó al consentimiento informado como la expresión máxima del principio de autonomía.
Respecto a la aplicación de los principios bioéticos, en el primer caso, destinado a reconocer la aplicación de la beneficencia y no maleficencia, un $27,8 \%(n=15)$ estuvo totalmente de acuerdo en la opción que resalta la aplicación de estos principios. En el segundo caso, únicamente el 37\% (n=20) demostró la aplicación de la no maleficencia; en el tercer caso aplicado en la encuesta, solo el 7,4\% ( $n=4)$ está totalmente en desacuerdo, resaltando la aplicación de la autonomía si se evalúa al principio dentro de un sistema óptimo de salud, mientras que el 83,8\% $(n=45)$ reflejó la aplicación del mismo principio en un sistema de salud en desarrollo. En el cuarto caso se analizó la aplicación del principio de justicia: el 55,6\% (n=30) de los encuestados demostró su aplicación, mientras que la beneficencia, en el quinto caso, se interpretó como aplicada por el 59,3\% ( $\mathrm{n}=32)$ de los participantes; de igual manera, la justicia en el sexto caso se aplica en un $51,9 \%(n=28)$.

Respecto de los participantes que recibieron la cátedra de bioética $(n=45)$ en su formación profesional, el 46,7\% $(\mathrm{n}=21)$ identificó los cuatros principios bioéticos; el 42,2\% ( $\mathrm{n}=19)$ señaló a la veracidad como el quinto principio de conducta del personal odontológico; únicamente el 11,1\% $(n=5)$ indicó que el Informe Belmont es un documento histórico que recalca los principios bioéticos; el 73,3\% ( $n=33)$ consideró al respeto por la dignidad humana como el principio más elevado en atención sanitaria; mientras que el $42,2 \%$ $(\mathrm{n}=19)$ señaló a la autonomía como representación del consentimiento informado. En el análisis de aplicación de principios bioéticos, el 31,1\% $(\mathrm{n}=14)$ reflejó la aplicación del principio de beneficencia y no maleficencia en el primer caso planteado; el 40\% ( $n=18)$ aplicó el principio de no maleficencia en el segundo caso; en el tercer caso, dentro de un sistema óptimo de salud, el $5,5 \%(\mathrm{n}=3)$ respetó la autonomía, pero el $84,4 \%$ $(\mathrm{n}=38)$ aplicó el mismo principio si se enfoca el caso a un sistema en desarrollo; el 55,6\% $(n=25)$ demostró aplicar en su práctica clínica la justicia; mientras que el 53,3\% ( $\mathrm{n}=24)$ reflejó actuar acorde a la beneficencia en el quinto caso $(\mathrm{P}<0.05)$ y el $46,7 \%(n=21)$ la autonomía y justicia en el sexto caso expuesto. 
Conocimiento y aplicación de los principios bioéticos en alumnos de posgrado de Odontología - Gabriela Morales et al.

Tabla 1. Correlación entre formación en bioética como cátedra de pregrado, variables de conocimiento y aplicación de principios bioéticos

\begin{tabular}{|c|c|c|c|c|c|}
\hline \multirow[t]{2}{*}{ Formación en bioética } & \multicolumn{2}{|c|}{$\begin{array}{l}\text { Como cátedra de pregrado } \\
(\mathrm{n}=45)\end{array}$} & \multicolumn{2}{|c|}{ Sin cátedra } & \multirow[t]{2}{*}{$\begin{array}{l}\text { Chi Cuadrado } \\
\text { de Pearson }\end{array}$} \\
\hline & Correcta & Incorrecta & Correcta & Incorrecta & \\
\hline \multirow[t]{2}{*}{ Reconoce los cuatro principios } & $46.7 \%$ & $53.3 \%$ & $22.2 \%$ & $77.8 \%$ & \multirow[t]{2}{*}{0.176} \\
\hline & $(\mathrm{n}=21)$ & $(n=21)$ & $(n=2)$ & $(n=7)$ & \\
\hline \multirow{2}{*}{$\begin{array}{l}\text { Reconoce el quinto principio } \\
\text { propuesto por la ADA }\end{array}$} & $42.2 \%$ & $57.8 \%$ & $33.3 \%$ & $66.7 \%$ & \multirow[t]{2}{*}{0.620} \\
\hline & $(\mathrm{n}=19)$ & $(n=26)$ & $(n=3)$ & $(n=6)$ & \\
\hline \multirow{2}{*}{$\begin{array}{l}\text { Reconoce el documento histórico } \\
\text { sobre principios bioéticos }\end{array}$} & $11.1 \%$ & $88.9 \%$ & $22.2 \%$ & $77.8 \%$ & \multirow[t]{2}{*}{0.365} \\
\hline & $(n=5)$ & $(n=40)$ & $(n=2)$ & $(n=7)$ & \\
\hline \multirow{2}{*}{$\begin{array}{l}\text { Reconoce el principio más elevado } \\
\text { en atención sanitaria }\end{array}$} & $73.3 \%$ & $26.7 \%$ & $55.6 \%$ & $44.4 \%$ & \multirow[t]{2}{*}{0.286} \\
\hline & $(\mathrm{n}=33)$ & $(\mathrm{n}=12)$ & $(n=5)$ & $(n=4)$ & \\
\hline \multirow{2}{*}{$\begin{array}{l}\text { Reconoce al consentimiento } \\
\text { informado como máxima expresión } \\
\text { del principio de autonomía }\end{array}$} & $42.2 \%$ & $57.8 \%$ & $33.3 \%$ & $66.7 \%$ & \multirow[t]{2}{*}{0.620} \\
\hline & $(n=19)$ & $(n=26)$ & $(n=3)$ & $(n=6)$ & \\
\hline \multirow{2}{*}{$\begin{array}{l}\text { Caso 1: Aplicación del principio de } \\
\text { beneficencia y no maleficencia }\end{array}$} & $31.1 \%$ & $68.9 \%$ & $11.1 \%$ & $88.9 \%$ & \multirow[t]{2}{*}{0.221} \\
\hline & $(n=14)$ & $(n=31)$ & $(n=1)$ & $(n=8)$ & \\
\hline \multirow{2}{*}{$\begin{array}{l}\text { Caso 2: Aplicación del principio de } \\
\text { no maleficencia }\end{array}$} & $40.0 \%$ & $60.0 \%$ & $37.0 \%$ & $77.8 \%$ & \multirow[t]{2}{*}{0.313} \\
\hline & $(\mathrm{n}=18)$ & $(\mathrm{n}=27)$ & $(n=2)$ & $(n=7)$ & \\
\hline \multirow{2}{*}{$\begin{array}{l}\text { Caso 3.1: aplicación del principio } \\
\text { de autonomía en un sistema de } \\
\text { salud óptimo }\end{array}$} & $6.7 \%$ & $93.3 \%$ & $11,1 \%$ & $88.9 \%$ & \multirow[t]{2}{*}{0.642} \\
\hline & $(\mathrm{n}=3)$ & $(\mathrm{n}=42)$ & $(n=1)$ & $(\mathrm{n}=8)$ & \\
\hline \multirow{2}{*}{$\begin{array}{l}\text { Caso 3.2: aplicación del principio } \\
\text { de autonomía en un sistema de en } \\
\text { desarrollo }\end{array}$} & $84.4 \%$ & $15.6 \%$ & $77.8 \%$ & $22.2 \%$ & \multirow[t]{2}{*}{0.624} \\
\hline & $(\mathrm{n}=38)$ & $(n=7)$ & $(n=7)$ & $(n=2)$ & \\
\hline \multirow{2}{*}{$\begin{array}{l}\text { Caso 4: aplicación del principio de } \\
\text { justicia }\end{array}$} & $55.6 \%$ & $44.4 \%$ & $55,6 \%$ & $44,4 \%$ & \multirow[t]{2}{*}{0.645} \\
\hline & $(n=25)$ & $(\mathrm{n}=20)$ & $(n=5)$ & $(n=4)$ & \\
\hline \multirow{2}{*}{$\begin{array}{l}\text { Caso 5: aplicación del principio de } \\
\text { beneficencia }\end{array}$} & $53.3 \%$ & $46.7 \%$ & $88.9 \%$ & $11.1 \%$ & \multirow[t]{2}{*}{${ }^{*} 0.048$} \\
\hline & $(n=24)$ & $(\mathrm{n}=21)$ & $(\mathrm{n}=8)$ & $(\mathrm{n}=1)$ & \\
\hline \multirow{2}{*}{$\begin{array}{l}\text { Caso 6: aplicación de los principios } \\
\text { de autonomía y justicia }\end{array}$} & $46.7 \%$ & $53.3 \%$ & $77.8 \%$ & $22.2 \%$ & \multirow[t]{2}{*}{0.088} \\
\hline & $(\mathrm{n}=21)$ & $(\mathrm{n}=24)$ & $(n=7)$ & $(\mathrm{n}=2)$ & \\
\hline
\end{tabular}

* Correlación.

El 7,4\% de participantes no respondió correctamente a ninguna de las preguntas de conocimiento sobre bioética.

Las encuestas reflejaron que el $40,7 \%$ de participantes tuvieron un conocimiento sobre principios bioéticos mayor al 60\%; mientras que en $11,1 \%$ su conocimiento fue mayor al $80 \%$.

De los participantes que tuvieron un conocimiento mayor al 60\% ( $n=22)$, el 40,9\% ( $n=9)$ aplicó en el primer caso propuesto los principios de beneficencia y no maleficencia; el 59,1\% ( $n=13)$ reflejó aplicar el principio de no maleficencia en el segundo caso ( $\mathrm{p}=0.005)$; en el tercer caso, ante un sistema óptimo de salud, el 4,5\% (n=1) aplicó el principio de autonomía, mientras que, ante un sistema en desarrollo, el principio fue aplicado por el $86,4 \%(n=19)$; en el cuarto caso, el $54,5 \%$ $(n=12)$ reveló actuar bajo el principio de justicia, en el quinto caso la beneficencia fue aplicada en un $50 \%(\mathrm{n}=11)$ y el $54,5 \%(\mathrm{n}=12)$ respetó el principio de autonomía y justicia en el sexto caso propuesto en el estudio.

Del grupo de participantes que presenta conocimiento mayor al $80 \%(n=6)$, el $50 \%(n=3)$ demostró aplicar el principio de beneficencia y no maleficencia en el primer caso; el 83,3\% ( $n=5)$ aplicó el principio de no maleficencia en el se- 
Tabla 2. Correlación entre conocimiento menor al $80 \%$ y mayor al $80 \%$ con relación a la aplicación de principios bioéticos

\begin{tabular}{|c|c|c|c|c|c|c|}
\hline \multirow{2}{*}{ Conocimiento } & \multicolumn{2}{|c|}{ Conocimiento menor al $80 \%^{1}$} & \multicolumn{2}{|c|}{ Conocimiento mayor al $80 \%{ }^{2}$} & \multirow{2}{*}{$\begin{array}{l}\text { Chi cuadrado } \\
\text { de Pearson }^{1}\end{array}$} & \multirow{2}{*}{$\begin{array}{l}\text { Chi cuadrado } \\
\text { de Pearson }{ }^{2}\end{array}$} \\
\hline & SI & $\mathrm{NO}$ & SI & $\mathrm{NO}$ & & \\
\hline \multirow{2}{*}{ CASO 1} & $40.9 \%$ & $59.1 \%$ & $50.0 \%$ & $50.0 \%$ & \multirow{2}{*}{0.074} & \multirow{2}{*}{0.197} \\
\hline & $(n=9)$ & $(n=13)$ & $(n=3)$ & $(n=3)$ & & \\
\hline \multirow{2}{*}{ CASO 2} & $59.1 \%$ & $40.9 \%$ & $83.3 \%$ & $16.7 \%$ & \multirow{2}{*}{${ }^{*} 0.005$} & \multirow{2}{*}{${ }^{*} 0.013$} \\
\hline & $(n=13)$ & $(\mathrm{n}=9)$ & $(n=5)$ & $(\mathrm{n}=1)$ & & \\
\hline \multirow{2}{*}{ CASO 3.1} & $4.5 \%$ & $95,5 \%$ & $0 \%$ & $100.0 \%$ & \multirow{2}{*}{0.506} & \multirow{2}{*}{0.462} \\
\hline & $(n=1)$ & $(\mathrm{n}=21)$ & $(\mathrm{n}=0)$ & $(n=6)$ & & \\
\hline \multirow{2}{*}{ CASO 3.2} & $86.4 \%$ & $13.6 \%$ & $83.3 \%$ & $16.7 \%$ & \multirow{2}{*}{0.620} & \multirow{2}{*}{0.640} \\
\hline & $(n=19)$ & $(n=3)$ & $(n=5)$ & $(\mathrm{n}=1)$ & & \\
\hline \multirow{2}{*}{ CASO 4} & $54.5 \%$ & $45.5 \%$ & $50.0 \%$ & $50.0 \%$ & \multirow{2}{*}{0.901} & \multirow{2}{*}{0.771} \\
\hline & $(n=12)$ & $(\mathrm{n}=10)$ & $(n=3)$ & $(n=3)$ & & \\
\hline \multirow{2}{*}{ CASO 5} & $50.0 \%$ & $50.0 \%$ & $33.3 \%$ & $66.7 \%$ & \multirow{2}{*}{0.251} & \multirow{2}{*}{0.170} \\
\hline & $(n=11)$ & $(n=11)$ & $(n=2)$ & $(n=4)$ & & \\
\hline \multirow{2}{*}{ CASO 6} & $54.5 \%$ & $45.5 \%$ & $100.0 \%$ & $0.0 \%$ & \multirow{2}{*}{0.743} & \multirow{2}{*}{${ }^{*} 0.012$} \\
\hline & $(n=12)$ & $(\mathrm{n}=10)$ & $(n=6)$ & $(\mathrm{n}=0)$ & & \\
\hline
\end{tabular}

* Correlaciones.

gundo ( $\mathrm{p}=0.022)$; en el caso tres, el principio de la autonomía fue aplicado en $0 \%$ en un sistema de salud óptimo y un $83,3 \%(n=5)$ en un sistema de salud en desarrollo; el 50,0\% ( $\mathrm{n}=3)$ demostró actuar bajo el principio de justicia en el cuarto caso; mientras que el 33,3\% (n=2) aplicó la beneficencia en el quinto caso y el $100 \%$ reflejó respeto por los principios de autonomía y justicia en el último caso.

\section{Discusión}

Desde el nacimiento de la odontología, el fundamento moral del aprendizaje se derivó de la sociedad y se difundió por ella a partir de los aprendices de odontólogos, quienes adquirieron una lealtad natural hacia sus "maestros" odontólogos en persona y durante su práctica. Luego, las convenciones de odontólogos tuvieron una influencia positiva en la moralidad, la identidad jurídica y la lealtad profesional de los dentistas durante el desarrollo ético de su profesión en el siglo XIX $(12,13)$.

Los odontólogos estan obligados a actuar principalmente en interés de los pacientes, a informar a los pacientes de manera completa y honesta, permitirles tomar sus propias decisiones de tratamiento y brindarles una atención competente que mejore continuamente(14). Ser odontólogo es adoptar un compromiso con la sociedad, aceptar las normas y obligaciones de la profesión, basadas en la búsqueda del bienestar mayor para el paciente(3). El odontólogo debe mantener un juicio último práctico para regular sus acciones; respetar las normas y guías de práctica clínica; mantener los principios bioéticos como guías de acción y conocer las teorías éticas que proporcionan justificación ética en la práctica clínica(15). Estos principios, de larga data en la práctica del cuidado de la salud, encontraron una nueva expresión en la "Carta de Profesionalismo Médico", que puede extenderse al área de la salud oral. Este documento es el trabajo de un consorcio internacional que ha revisado la aplicabilidad de los estándares éticos existentes a los desafíos que enfrentan los profesionales del siglo XXI, y ha reafirmado los principios fundamentales de la práctica ética del cuidado de la salud para llevar a cabo un código de conducta para el nuevo siglo(14).

En el mundo, el desarrollo de la educación ética de los profesionales de la salud oral se manifestó desde principios de la década de 1980, con planes de estudio informales que a menudo eran incorporados a cursos de jurisprudencia o de gestión práctica, y con pocos materiales educativos eficaces. Durante la década siguiente, la Asociación Estadounidense de Escuelas de Odontología 
aprobó directrices formales para la enseñanza de ética. Asimismo, aparecieron varios textos y revistas con columnas de ética y se creó la Red de Ética Profesional en Odontología(16). En la actualidad, la formación en bioética ha sido reconocida como estrictamente necesaria en la competencia del profesional de salud, teniendo como objetivo proporcionar elementos de juicio que contribuyan a la comprensión de dilemas y conflictos que se presenten durante la práctica profesional(17). El siguiente paso fue desarrollar un foro regular para la publicación de artículos sobre ética en el cuidado dental(16). Por este motivo, la Organización Panamericana de la Salud (OPS) contribuye en el campo odontológico, desde 2005, con la realización de "Jornadas de Bioética en Odontología” (18); procurando concientizar a los expertos del cuidado de salud oral en los distintos temas que puedan brindarle las herramientas necesarias al momento de tomar decisiones.

En Ecuador, para este gremio, se ha presentado la "Declaración de Quito sobre Bioética y Salud Oral", de 2008, en la que se rectificó la necesidad de promover la enseñanza bioética en todos los niveles de educación(11). Sin embargo, este estudio evidenció que el 16,7\% de los encuestados afirmó no haber recibido la cátedra de bioética dentro de su formación de pregrado. Por otro lado, a pesar de ser un deber profesional mantenerse en constante actualización, únicamente, el $7,4 \%$ de los participantes ha recibido clases formales en el tema. Para Aranguren, la bioética en la odontología ha tenido una lenta incorporación debido a que no abarca los grandes y controversiales temas que la rodean(19). Sin embargo, esto no significa que sea ajena al gremio la búsqueda del aprendizaje permanente en un contexto de responsabilidad ética y social de los profesionales de la salud(20). Además, requiere la aplicación de metodologías dirigidas a la reflexión, que animen a los estudiantes de odontología a desarrollar habilidades para analizar una estrategia eficaz de atención, en la que la ética se convierta en un valor cardinal $(21,22)$.

Únicamente el 19,6\% de la población identificó al CEEDO; coincidiendo con el estudio realizado por Pachacama-Palacios en el 2017(23); en el que se mencionó que el conocimiento sobre este código es regular.
En cuanto a las preguntas relacionadas con el conocimiento sobre los principios bioéticos, el $57,4 \%$ no reconoció la autonomía, justicia, beneficencia y no maleficencia como principios bioéticos, a pesar de ser la base fundamental del CEEDO(11), porcentaje mayoritario justificado con el desconocimiento sobre el mismo; el $59,3 \%$ identificó a la veracidad como principio de conducta del profesional odontológico, según la Asociación Dental Americana, inmersa en este código desde el 2016, y reafirmada en su última actualización realizada en el 2020(24). El 87\% desconoce al Informe Belmont como documento que destacó los principios bioéticos y las pautas para la protección de seres humanos. Sin embargo, el 70,4\% de los participantes señaló que el respeto por la dignidad humana es el principio bioético más elevado, basado en el enfoque integral y humanista en la asistencia sanitaria; los profesionales demostraron estar conscientes de que este principio debe ser aplicado a la atención odontológica y a las relaciones interpersonales odontólogo-paciente, odontólogo-equipo de trabajo y odontólogo-comunidad(24).

Respecto del consentimiento informado, y a pesar de ser un requisito de la práctica sanitaria y un derecho del paciente, el 59,3\% de los profesionales encuestados no lo relacionó con el principio de autonomía. El estudio realizado por Palomer determina que un gran porcentaje de odontólogos aplican este instrumento con el objetivo de evitar problemas legales(25).

Se dio a conocer a los participantes seis casos clínicos en los que se buscó identificar los principios bioéticos inmersos. En el primer caso, el 27,8\% de los participantes seleccionó actuar según los principios de beneficencia y no maleficencia, cumpliendo con el deber del profesional odontológico de conocer sus debilidades y actuar dentro de su competencia(11). En el segundo caso, el 37\% de los encuestados se apegó a las acciones que resaltan al principio de no maleficencia, procurando la derivación del paciente al especialista(24).

El tercer caso fue direccionado a dos escenarios, proyectándolo dentro de un sistema óptimo de salud, en el que se esperaría que se realicen todos los procesos y tratamientos prudentes para evitar la pérdida dental. Respecto de este escena- 
rio, el 7,4\% de los encuestados dijo respetar la autonomía del paciente, evitando a toda costa la exodoncia o rechazando realizar el tratamiento; mientras que, enfocando el caso a un segundo escenario, con un sistema de salud en desarrollo, el 83,3\% señaló respetar el principio de autonomía del paciente, justificando la exodoncia mediante la adquisición previa del respectivo consentimiento informado. El segundo escenario de este caso reflejó la realidad actual de la odontología dentro del sistema nacional de salud, demostrando la necesidad de educar a los futuros profesionales para que sean miembros competentes del equipo interprofesional de salud en el que se fomente el bienestar del paciente(26).

El cuarto caso, destinado a reconocer el accionar justo entre profesionales, demostró que el 55,6\% aplicó el principio de justicia en cuanto a la aceptación de pacientes, y el respeto a la relación entre $\operatorname{colegas}(6,11)$.

En el quinto caso planteado, el 59,3\% de los odontólogos aplicó el principio de beneficencia, procurando el bien mayor para el paciente vulnerable(27). En el sexto caso, estructurado para identificar la aplicación de los principios de justicia y autonomía, el 51,9\% de los participantes obtuvieron resultados compatibles con el respeto a la autoridad de los tutores; los odontólogos reconocieron que la familia y los tutores son la primera red de apoyo y quienes toman las decisiones y proporcionan el consentimiento informado de los pacientes más vulnerables(28).

Respecto de la relación entre la formación en bioética como cátedra, dentro de la formación de pregrado, a pesar de que el $83,3 \%$ de los encuestados afirmó haber recibido la cátedra dentro de su formación de pregrado, no reflejó una relación entre estos estudios y el conocimiento sobre principios bioéticos. Esto podría deberse, como menciona Torres-Quintana(3), a que las instituciones de educación dental cumplen con su compromiso de formar profesionales más éticos y humanos; sin embargo, no cuentan con docentes cuyo único o principal objetivo sea impartir ética odontológica. Muchas veces el contenido del curso es inadecuuado, porque no fomenta una base introspectiva para generar un cambio de comportamiento $(3,29)$. Entender la ética dental como un campo independiente de la ética medica es un enfoque necesario en bioética, que se siente particularmente durante la formación del profesional. Se requiere establecer un plan de estudio específico que prepare para enfrentar los desafíos éticos durante la práctica profesional(30). Por otro lado, en la aplicación de principios bioéticos en la práctica clínica se mostró relación entre haber recibido la cátedra de bioética y procurar actuar bajo el principio de beneficencia.

No se reflejó relación entre los participantes que tomaron algún curso formal respecto a bioética respecto de sus conocimientos y la aplicación de principios bioéticos dentro de su práctica profesional; nadie ha elegido nunca hacer lo correcto por tomar un curso de ética(31).

El estudio realizado por Pachacama-Palacios hace referencia al documento "Normas y Procedimientos de Atención en Salud Bucal Primer Nivel del Ministerio de Salud Pública", señalando que "es pertinente que se realice orientación y educación en cuestiones de Ética y Bioética, y ayude a concretar políticas y acciones en esta materia a todo el personal odontológico"(23,32). Los resultados en este estudio resaltaron la importancia del enunciado, ya que el 7,4\% de los participantes no reflejó ningún nivel de conocimiento respecto de los principios bioéticos, mientras que el 29,6\% sí demostró conocer un $60 \%$, y un $11,1 \%$ un nivel de $80 \%$ de conocimiento sobre el tema, denotando la progresiva integración de la bioética en el campo de la odontología, como lo mencionaba Aranguren(19).

La correlación entre los participantes que mostraron los porcentajes mayoritarios de conocimiento y la aplicación de los principios bioéticos en la práctica odontológica determinó que aquellos que conocen entre $60 \%$ y $80 \%$ sobre el tema, aplicaron el principio de no maleficencia, procurando obrar en favor de mejorar la calidad de vida de los pacientes. Concordando con la necesidad señalada por Chavez, cuando menciona que es imperativo que los escenarios para reconocer, analizar y discutir cuestiones éticas estén integrados durante todo el proceso educativo del estudiante de odontología(33). 


\section{Conclusiones}

Se analizó cinco niveles de conocimiento, siendo los más relevantes los grupos que poseen conocimiento sobre los principios bioéticos entre el $60 \%$ y el $80 \%$, correspondiendo al $40,7 \%$ de los participantes que manifestaron, según el análisis realizado, la aplicación clínica de los principios de beneficencia y no maleficencia dentro de su práctica sanitaria.

En cuanto a los conocimientos de los principios bioéticos, se ha revelado el desconocimiento de la teoría; muchos de los participantes no conocen el CEEDO y los principios bioéticos propuestos como la base del correcto accionar del profesional de salud oral. Sin embargo, se ha demostrado un creciente cambio al respecto a la aplicación de estos principios.

Al analizar la aplicación de los principios bioéticos frente a los casos propuestos, podemos resaltar que el personal odontológico que ha recibido bioética en su formación de pregrado demuestra aplicar en su práctica clínica el principio de beneficencia; mientras que aquellos que se encuentran además en los más altos niveles de conocimiento también han aplicado el principio de no maleficencia. Lo que refleja no solo la presencia del perfil profesional que se espera del odontólogo, sino también el pausado, pero no ausente, crecimiento de la bioética en el área de salud bucodental.

La sombra del paternalismo acompaña a la práctica odontológica, debido al grado de conocimiento especializado relacionado con las enfermedades. El profesional debe ser capaz de explicar el procedimiento de manera sencilla, establecer una comunicación asertiva y enfocar su conocimiento científico para proponer diferentes soluciones. Por este motivo, es necesario que la catedra de bioética en la formación de personal de salud oral sea impartida de manera integral, procurando invitar al odontólogo a la reflexión tras la práctica clinica. Se recomienda que sea impartida de forma novedosa, contribuyendo a captar la atención e interés del estudiante para que mantenga una práctica consciente, enfocada en el respeto de la dignidad de sus pacientes, bajo la aplicación de los principios bioéticos. 


\section{Referencias}

1. Monzon JE, Ariasgago OL, Monzon-Wyngaard A. Assessment of moral judgment of BME and other health sciences students. In: Annual International Conference of the IEEE Engineering in Medicine and Biology Society IEEE Engineering in Medicine and Biology Society Annual International Conference; 2010: 2963-2966.

2. Castillo D V, Guerra A. Bioética desde una perspectiva odontológica. Estomatologia 2009 Dec 1; 17(2): 45-52.

3. Torres-Quintana MA, Romo OF. Bioética y ejercicio profesional de la odontología. Acta Bioethica 2006; 12(1): 65-74.

4. Izzeddin Abou R, Spina M, Tosta E. Dimensión ética en la práctica odontológica. Visión personalista. Acta Bioethica 2010; 16(2): 207-210.

5. Izzeddin Abou R, Jiménez F. Bioethics in Dentistry, a principled vision. Rev CES Odontol [Internet]; 2013 [cited 2020 Mar 31]; 26(1): 68-76. Available from: http://www.scielo.org.co/scielo.php?script=sci_arttext\&pid=S0120-971X2013 $000100007 \& \operatorname{lng}=$ en\&nrm=iso\&tlng=es

6. American Dental Association. Principle of Ethics \& Code of Professional Conduct. Am Dent Assoc [Internet] 2018; (November): 23. Available from: https://www.ada.org/ /media/ADA/Member Center/Ethics/Code_Of_Ethics_Book_ With Advisory Opinions Revised to November 2018.pdf?la=en

7. Arturo F, Salazar R. Bioética y veracidad. Cuando la palabra del médico puede llegar a profundizar el desvaliamiento del paciente. Acta Bioethica 2020; 26(1). Available from: https://orcid.org/0000-0003-

8. Kretschmann Ramírez R, Arenas Massa Á. Enseñanza de bioética en la carrera de odontología. Reflexiones y prospectivas. Pers y Bioética 2016; 20(2): 257-270.

9. Amorim AG, de Souza ECF. Problemas éticos vivenciados por dentistas: Dialogando com a bioética para ampliar o olhar sobre o cotidiano da prática profissional. Cienc e Saude Coletiva 2010 May; 15(3): 869-878.

10. Lolas Stepke F. Salud oral y bioética. Acta Bioethica 2006 Jan; 12(1): 7-8.

11. Gonzales M. Código Ecuatoriano de Ética y Deontología Odontológica. Quito, Ecuador: Editorial Grafamaranta; 2009.

12. Bishop MG, Gibbons DGS. Ethics; "in consideration of the love he bears." Apprenticeship in the nineteenth century, and the development of professional ethics in dentistry. Part 1. The practical reality 2002; 14(193).

13. MG B, D G, S G. Ethics; "In consideration of the love he bears." Apprenticeship in the nineteenth century, and the development of professional ethics in dentistry. Part 2. Hippocrates' long shadow. Br Dent J [Internet] 2002 Sep 28 [cited 2021 Jul 25]; 193(6): 321-325. Available from: https://pubmed.ncbi.nlm.nih.gov/12368888/

14. AL Y. Dental ethics for the 21st century: learning from the Charter on Medical Professionalism. J Am Coll Dent [Internet] 2002 Jan 1 [cited 2021 Jul 25]; 69(3): 53-60. Available from: https://europepmc.org/article/MED/12407998

15. Aramini M. Orígenes de la bioética y método de los principios. In: Introducción a la Bioética. 2nd ed. Bogotá, Colombia: San Pablo; 2007: 17-27.

16. Ozar DT. Dental ethics as an intellectual discipline: taking the next step. J Am Coll Dent 2000 Spring; 67(1): 30-34. PMID: 10812874. https://pubmed.ncbi.nlm.nih.gov/10812874/

17. León F. Enseñar Bioética: Cómo transmitir conocimientos, valores y actitudes. Acta Bioethica 2008; 14(1): 11-18.

18. Zaror Sánchez C, Muñoz Millán P, Espinoza Espinoza G, Vergara González C, Valdés García P. Enseñanza de la bioética en el currículo de las carreras de odontología desde la perspectiva de los estudiantes. Acta Bioethica 2014; 20(1): 135142.

19. Aranguren Ibarra ZM. Ejercer la Odontología desde una perspectiva bioética. Acta Odontológica Venez [Internet] 2014 [cited 2020 Mar 31]; 52(1): 1-12. Available from: https://www.actaodontologica.com/ediciones/2014/1/art-4/

20. Hobdell M, Sinkford J, Alexander C, Alexander D, Corbet E, Douglas C, Katrova L, Littleton P, MacCarthy D, Cherrett HM, Schou L, Wen FM, Zhuan B. Ethics, equity and global responsibilities in oral health and disease. Eur J Dent Educ 2002; 6 Suppl 3: 167-178. DOI: 10.1034/j.1600-0579.6.s3.23.x. PMID: 12390275. https://pubmed.ncbi.nlm. nih.gov/12390275/

21. Pirnay P. Faut-il enseigner la bioéthique aux étudiants en odontologie dans le cadre de la discipline santé publique ? Une expérience à la faculté de chirurgie dentaire de l'université Paris Descartes. Rev Epidemiol Sante Publique 2015 Jun $1 ; 63(3): 203-210$.

22. Garbin CAS, Gonçalves PE, Garbin AJI, Moimaz SAS. Evaluación de las metodologías de enseñanza-aprendizaje de la bioética en las facultades de odontología brasileñas. Rev la Fund Educ Médica 2009; 12(4): 231.

23. Pachacama G, Palacios E. Nivel de conocimiento y aplicación de los valores éticos y morales en la práctica profesional odontológica. Estudio referencial: capitales provinciales de la sierra ecuatoriana. Quito; 2017.

24. American Dental Association. Principles of ethics \& code of professional conduct. 2020 (November)

25. Palomer R. L. Consentimiento informado en odontología. un análisis teórico-práctico. Acta Bioethica [Internet] 2009 [cited 2021 Jun 9]; 15(1): 100-105. Available from: http://www.scielo.cl/scielo.php?script=sci_arttext\&pid=S1726- 
Conocimiento y aplicación de los principios bioéticos en alumnos de posgrado de Odontología - Gabriela Morales et al.

569X2009000100013\&lng=es\&nrm=iso\&tlng=es

26. Cino K, Austin R, Casa C, Nebocat C, Spencer A. Interprofessional ethics education seminar for undergraduate health science students: A pilot study. J Interprof Care 2018 Mar; 32(2): 239-241. DOI: 10.1080/13561820.2017.1387771. Epub 2017 Nov 9. PMID: 29120252. https://pubmed.ncbi.nlm.nih.gov/29120252/

27. De Vecchio Puplaksis N, Pereira da Silva MM, Nobile R, De Paula Ramos DL. A disciplina de bioética na Faculdade de Odontologia da Universidade de São Paulo. Rev Latinoam Bioética [Internet] 2010 [cited 2020 Mar 31]; 10(19): 68-75. Available from: http://www.scielo.org.co/scielo.php?pid=S1657-47022010000200006\&script=sci_abstract\&tlng=pt

28. Díaz Cárdenas S, Tirado Amador LR, Vidal Madera AM. Odontología con enfoque en salud familiar. Rev Cuba Salud Pública [Internet] 2014 [cited 2021 May 30]; 40(3): 397-405. Available from: http://scielo.sld.cu/scielo.php?script=sci_ arttext\&pid=S0864-34662014000300010

29. Bertolami CN. Why our ethics curricula don't work. J Dent Educ 2004 Apr; 68(4): 414-425. PMID: 15112918. Available from: https://pubmed.ncbi.nlm.nih.gov/15112918/

30. Gorkey S, Guven T, Sert G. Towards a specific approach to education in dental ethics: a proposal for organising the topics of biomedical ethics for dental education. J Med Ethics 2012 Jan; 38(1): 60-63. DOI: 10.1136/medethics-2011-100067. Epub 2011 Sep 2. PMID: 21890860. Available from: https://pubmed.ncbi.nlm.nih.gov/21890860/

31. Jenson LE. Why our ethics curricula do work. J Dent Educ 2005 Feb; 69(2): 225-228; discussion 229-32. PMID: 15689605.

32. Ministerio de Salud Pública del Ecuador. Normas y procedimientos de atención en Salud bucal en el primer nivel: 2009.

33. Chaves JF. Assessing ethics and professionalism in dental education. J Indiana Dent Assoc 2000 Spring; 79(1): 16-21. PMID: 11314371

Recibido: 11 de agosto de 2021

Aceptado: 16 de septiembre de 2021 